RANA 03-04

February 2003

Periodic AC and Periodic Noise in RF Simulation for Electronic Circuit Design

by

E.J.W. ter Maten, F.G. Fijnvandraat,

C. Lin and J.M.F. Peters

Reports on Applied and Numerical Analysis

Department of Mathematics and Computer Science

Eindhoven University of Technology

P.O. Box 513

$5600 \mathrm{MB}$ Eindhoven, The Netherlands

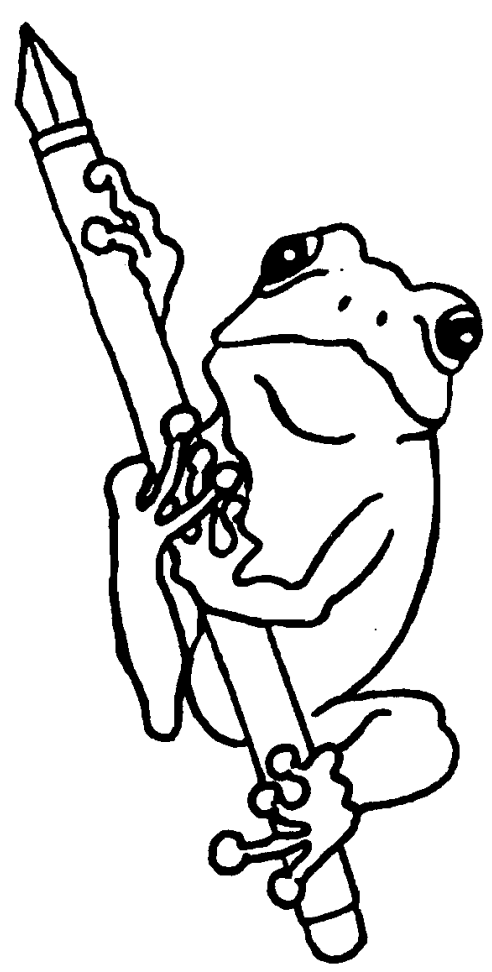

ISSN: 0926-4507 


\title{
Periodic AC and Periodic Noise in RF Simulation for Electronic Circuit Design
}

\author{
E.J.W. ter Maten, J.G. Fijnvandraat, C. Lin, and J.M.F. Peters
}

\begin{abstract}
This overview highlights the main steps to come to Periodic AC and Periodic Noise simulation in RF simulation. A distinction is made between forced oscillators and free oscillators. Noisy elements contribute in various ways to the overall noise effect. When dealing with free oscillators, phase noise is most important. In the case of white and coloured stochastic noise sources, the spectrum of the (stationary) autocorrelation of the phase shifted function can be determined by summing the effects of all independent noise sources. In general one has to take the non-linear way, in which the phase shift function changes with time, into account.
\end{abstract}

\section{RF simulation methods}

The newly developed simulation methods for radio frequency (RF) problems all somehow exploit the 'sparsity' of the signal spectra. The basic method is that of determining the (time) periodic steady-state (PSS) solution of a circuit. Conceptually this can be seen as a generalisation of the well-known DC operating point (the steady-state or Direct Current solution): for baseband circuits the spectral content around $0 \mathrm{~Hz}$ (the DC point) is important. For RF circuits the (narrow) spectral content around specific frequencies (of the PSS solution) is of interest. This PSS solution can be obtained in the frequency domain (e.g. by applying the harmonic balance method $[16,17]$ ) or in the time domain (by methods, like shooting, based on transient simulation $[7,9,10,13,18])$. With baseband simulation, after determining the $\mathrm{DC}$ point, additional simulations like AC, noise, etc. can be done to obtain more information about the circuit. Similarly, based on the PSS solution several other simulations can be done like periodic $\mathrm{AC}$, periodic noise, etc., $[1,19,20,21,22]$. In view of the $\mathrm{RF}$ circuit and signal characteristics, the PSS solution determines the non-linear behaviour of the circuit while the periodic $\mathrm{AC}$, etc. deals with the frequency shift.

The main difference between the time domain and frequency domain methods to obtain the PSS solution is that the former can easily deal with strongly non-linear

Received by the editors December 15, 2002.

1991 Mathematics Subject Classification. Primary 65L10; Secondary 65L80.

Key words and phrases. circuit simulation, radio frequency, RF, periodic steady state, PSS, periodic AC, periodic noise, phase noise, perturbation analysis, linear time-varying, LTV, differentialalgebraic equations, autonomous systems, forced oscillators, free oscillators. 
circuits and discontinuities and have good convergence properties while the latter deal naturally with components characterised in the frequency domain. Over the years combinations of both basic methods were developed resulting in mixed timefrequency domain approaches each with their own advantages and drawbacks.

A two-step approach appears to be powerful as well as practical for simulating $R F$ mixing noise:

- Determine the noiseless Periodic Steady-State (PSS) solution as largesignal solution. This can be done in the time domain, the frequency domain or by using mixed time-frequency methods. The time-domain representation is a time-varying solution.

Of course, a noiseless PSS analysis (with or without determining the oscillation frequency), has value on its own for RF simulations.

- Apply a linearisation around the PSS solution and study noise as a small signal perturbation. The noise sources may contain frequencies that are different from the PSS solution.

For simulating RF phase noise or timing jitter (i.e. shifts in zero crossings of the solution) of free oscillators, applying as second step a linearisation around the PSS solution and study noise as small signal perturbations is of limited use [4]. In fact, the results are only useful for small $t$, because the resulting deviations may grow large with time.

The non-linear perturbation analysis, proposed in [4], is an alternative to the second step. Also in this approach, the first step is necessary. The non-linear perturbation analysis results in a correct phase deviation. For the orbital deviation, again a linearisation around the PSS solution (but including phase deviation) can be used. This implies that periodicity of the coefficients of the linear time-varying differential equation can not be assumed. It also implies that, in general, the phase deviation is a time-varying function.

This paper shortly describes the PSS problem and concentrates on aspects for RF $\mathrm{AC}$ and RF Noise analysis.

\section{The PSS Problem}

The Periodic Steady-State (PSS) problem for one overall period $T>0$ is defined as:

$$
\begin{aligned}
\frac{\mathrm{d}}{\mathrm{d} t} q(t, x)+j(t, x) & =0 \in \mathbf{R}^{N} \\
x(0)-x(T) & =0
\end{aligned}
$$

where $q(t, x)$ denotes charges (assembled at the respective nodes) and fluxes, and where $j(t, x)$ covers the static part (and sources as well). In general (2.1) is a system of Differential-Algebraic Equations (DAE). Clearly, for all $t \in \mathbb{R}, x(t)=x(t+T)$. A function $x: \mathbb{R} \rightarrow \mathbb{R}^{n}$ is called a Periodic Steady-State Solution if there is a $T>0$ such that $x$ satisfies (2.1)-(2.2). Note that according to this definition, a stationary solution (called the DC, direct current, solution), i.e. a solution of the 
form $x(t) \equiv x_{0}$, is also a PSS solution.

To define the PSS problem precisely, we have to introduce the concept of limit cycles and define stability for PSS solutions and limit cycles.

The limit cycle $\mathcal{C}(x)$ of a PSS solution $x$ is the range of the function $x(t)$, i.e.

$$
\mathcal{C}(x)=\{x(t) \mid t \in \mathbb{R}\} .
$$

A set $\mathcal{C}$ is called a limit cycle of (2.1) if there is a PSS solution $x$ of $(2.1)$ so that $\mathcal{C}=\mathcal{C}(x)$.

A PSS solution $x$ is called stable (some authors prefer the term strongly stable) if there is a $\delta>0$ such that for every solution $x^{*}$ of (2.1) that has the property

$$
\exists_{\tau_{1}>0}\left\|x^{*}(0)-x\left(\tau_{1}\right)\right\|<\delta
$$

there exists a $\tau_{2}>0$ such that

$$
\lim _{t \rightarrow \infty}\left\|x^{*}(t)-x\left(t+\tau_{2}\right)\right\|=0 .
$$

A limit cycle is called stable when all of its periodic steady states are stable.

Periodic steady-state solutions that are not stable are not of interest to the IC designer, since they do not correspond to any physical behaviour of the modelled circuit. In fact, we want to actively avoid non-stable periodic steady-state solutions for this reason.

An exception to the above might be the DC solution, which, in the free oscillating case, is the most well-known unstable solution. Also numerically the DC solution is of interest because it provides a way to find (approximate, initial) periodically time-varying stable solutions, by perturbing the DC solution.

For forced, or driven, (i.e. non-autonomous) problems all explicit time-dependent coefficients and sources are periodic with a common (known) period $T$. When dealing with autonomous circuits (also called free-running oscillator circuits) the functions $q$ and $j$ do not explicitly depend on time and $j$ does not have timedependent external sources. The PSS problem is defined by

$$
\begin{aligned}
\frac{\mathrm{d}}{\mathrm{d} t} q(x)+j(x) & =0 \in \mathbb{R}^{N}, \\
x(0)-x(T) & =0 .
\end{aligned}
$$

In spite of this, a time-varying periodic steady-state solution may exist for some particular value of $T$. We will call this solution the oscillation solution and $\omega_{\text {osc }}$ and $f_{\mathrm{osc}}$, given by $\omega_{\mathrm{osc}}=2 \pi f_{\mathrm{osc}}=\frac{2 \pi}{T}$, the angular and 'normal' oscillation frequency, respectively. In the autonomous case, solution and oscillation frequency have to be determined both. Mathematically, the problem is a non-linear eigenproblem. In the autonomous case, it is clear that when $x(t)$ is a solution of (2.6), another 
solution can simply be constructed by making a time shift: $\tilde{x}(t)=x\left(t-t_{0}\right)$. To make the problem unique, in practice one gauges the solution by requiring that

$$
e_{i}^{\top} x\left(t_{0}\right)=c
$$

(for some coordinate $i$ and constant $c$ ) [clearly $c$ should be determined in the range of $x$, but not equal to a DC-value], or by imposing a condition on the timederivative

$$
e_{i}^{\top} x^{\prime}\left(t_{0}\right)=c
$$

Now the system (2.6) and (2.7), resp. (2.8), defines a non-linear problem with a "unique" solution for the unknowns $x, T$ : small time shifts are excluded.

Rescaling the time by writing $t=s T$, with $s \in[0,1]$, we have

$$
\begin{aligned}
\frac{\mathrm{d}}{\mathrm{d} t} q(x(t))+j(x(t)) & =\frac{1}{T} \frac{\mathrm{d}}{\mathrm{d} s} q(x(s T))+j(x(s T)) \\
& =\frac{1}{T} \frac{\mathrm{d}}{\mathrm{d} s} q(\hat{x}(s))+j(\hat{x}(s)),
\end{aligned}
$$

where $\hat{x}(s)=x(s T)$. Note that $\hat{x}(1)=x(T)$. Hence, the problem (2.6) can also be studied on the unit interval for the function $\hat{x}(s)$ after scaling the $s$-derivative by a factor $1 / T$.

In fact, $T$ can be nicely added to the system as well

$$
\begin{aligned}
\frac{1}{T} \frac{\mathrm{d}}{\mathrm{d} s} q(\hat{x}(s))+j(\hat{x}(s)) & =0 \\
\frac{\mathrm{d}}{\mathrm{d} s} T & =0 \\
\hat{x}(0) & =\hat{x}(1) \\
e_{i}^{\top} \hat{x}(0) & =c
\end{aligned}
$$

(Clearly, $T$ automatically fulfills the periodicity condition).

\section{Perturbation analysis}

We assume that the PSS solution has been determined by some numerical procedure $[7,9,10,13,18]$. In this section we will consider the problem for a subsequent perturbation analysis. The PSS solution of $(2.1)$ will be denoted by $x_{P S S}$. It will also be called the noiseless time-varying large signal solution. Now we perturb the left-hand side of (2.1) by adding some small (noise) function $n$

$$
\frac{\mathrm{d}}{\mathrm{d} t} q(t, x)+j(t, x)+n(t)=0 \in \mathbf{R}^{N},
$$

which results in a solution of the form

$$
x(t)=x_{P S S}(t+\alpha(t))+x_{n}(t),
$$

where the phase shift function $\alpha(t)$ still has to be prescribed and $x_{n}(t)$ remains small. 


\subsection{Linear perturbation analysis for forced systems}

Linearising (3.1) around $x_{P S S}$ (i.e. considering the case $\alpha(t)=0$ ), results in a Linear Time-Varying (LTV) differential equation for $x_{n}$

$$
\begin{aligned}
\frac{\mathrm{d}}{\mathrm{d} t}\left(C(t) x_{n}\right)+G(t) x_{n}+n(t) & =0 \in \mathbb{R}^{N} \\
C(t)=\left.\frac{\partial q(t, x)}{\partial x}\right|_{x_{\mathrm{PSS}}}, \quad G(t) & =\left.\frac{\partial j(t, x)}{\partial x}\right|_{x_{\mathrm{PSS}}} .
\end{aligned}
$$

In practical applications, a basic noise term has the form

$$
\begin{aligned}
& n(t)=B(t) b(t), \\
& B(t)=B\left(x_{\mathrm{PSS}}(t)\right),
\end{aligned}
$$

that consists of a normalized (scalar) perturbation function $b(t)$, that is modulated by the periodic (vector) function $B(t)=B\left(x_{P S S}(t)\right)$. Here $b(t)$ may be defined most conveniently in the frequency domain, while $B\left(x_{P S S}(t)\right)$ is defined by expressions in the time domain. The validity of this approach was established in [4]. In noise simulations, the noise sources are included automatically by placing current sources parallel to the noisy element, which action does not disturb the topology. For forced systems the perturbed solution $x(t)$ can be approximated by (3.2) with $\alpha$ being identically zero and $x_{n}$ the solution of (3.3). However, when dealing with free oscillators a non-trivial choice for the phase-shift function $\alpha(t)$ has to be made. We note that the coefficients in (3.3) are periodic in $t$ with period $T$. Thus, they can be expanded in exponentials $e^{i \omega_{k} t}$, in which $\omega_{k}=2 \pi k / T$. It is instructive to consider the case for a simple sine-wave source, i.e. when

$$
n(t)=U e^{i \nu t}
$$

in which $U$ does not depend on time, and $\nu=2 \pi f_{n} ; f_{n}$ may be interpreted as a noise frequency. Here $\nu$ may be different from $\omega_{k}$. Introducing $y_{n}(t) \equiv e^{-i \nu t} x_{n}(t)$ results in a linear DAE of which source term and (complex) coefficients (that depend on the parameter $\nu$ ) are periodical with period $T$

$$
\frac{\mathrm{d}}{\mathrm{d} t}\left(C(t) y_{n}\right)+[G(t)+i \nu C(t)] y_{n}+U=0 \in \mathbb{R}^{N}
$$

When $x_{P S S}(t) \equiv x_{D C}$, and $[G(t)+i \nu C(t)]$ is regular (and time-independent), the solution $y_{n}$ is time-independent and simply equals the well-known AC solution. For the general case, we find that $y_{n}$ and $x_{n}$ have expansions of the form (see also $[20,21])$

$$
\begin{aligned}
& y_{n}(t)=\sum_{k=-\infty}^{\infty} y_{n, k}^{(\nu)} e^{i \omega_{k} t} \\
& x_{n}(t)=\sum_{k=-\infty}^{\infty} y_{n, k}^{(\nu)} e^{i\left(\nu+\omega_{k}\right) t} .
\end{aligned}
$$


Because of the periodic coefficients in (3.3) and (3.8), the determination of the $y_{n, k}^{(\nu)}$ is called Periodic AC (PAC) analysis. The expansion of $x_{n}(t)$ implies that

$$
\begin{aligned}
x_{n}(t+T) & =\beta(\nu) x_{n}(t), \text { or } \\
x_{n}(0) & =\beta(-\nu) x_{n}(T), \text { where } \\
\beta(\nu) & =e^{i \nu T} .
\end{aligned}
$$

It is clear that, for a single input frequency $\nu$, the solution $x_{n}(t)$ contains frequencies of the form $\left(\nu+\omega_{k}\right)$, i.e. frequency folding occurs. In practice one considers so-called wide-band input signals with input frequencies $\left(\nu+\omega_{m}\right)$. If we allow for several input frequencies $\nu_{i}$, we can also say that a certain output frequency might originate from a large number of possible input frequencies. Hence, noise components at a certain frequency might end up in a different frequency band. This is why, for example, $1 / f$ noise which has its main energy at low frequencies, still plays an important role in RF circuits.

It is important to note that we described a linear perturbation analysis and we will not find contributions containing for example $\left(\nu_{1}+\nu_{2}+\omega_{k}\right),\left(\nu_{1}+2 \nu_{2}+\omega_{k}\right)$ etc. This assumption is in general not a severe limitation when simulating noise in RF circuits.

In $[20,21]$ one considers the integration of $(3.3)$ in which case the factor $\beta$ easily allows adaptive re-usage of linear algebra used for solving the PSS problem (see also [2]). However, the integration of (3.8) gives rise to even more elegant algorithms. Note that now the result for $x$ is obtained by an exact factor $e^{i \nu t}$.

\subsection{Perturbation analysis for free oscillating systems}

When dealing with perturbed oscillatory systems

$$
\frac{\mathrm{d}}{\mathrm{d} t} q(x)+j(x)+n(t)=0 \in \mathbb{R}^{N},
$$

it is no longer possible to assume that small perturbations $n(t)$ lead to small deviations in $x p S S(t)$ [An instructive example is provided by considering $y^{\prime}(t)+$ $\cos (t) y(t)-1=0$, of which the inhomogeneous solution is not periodic at all; however, note that $y(t+2 \pi)$ still satisfies the differential equation]. The main reason is that the period of the large signal solution is influenced by $n(t)$. This can lead to large (momentary) frequency deviations such that the difference between the noiseless and noisy solution (at the same time $t$ ) can no longer be considered to be small.

3.2.1. FlOQUeT THEORY We start with the necessary background of Floquet Theory when applied to oscillatory problems and which provides a way to a proper perturbation approach $[4,5,14,15]$. We start by noting that $x_{\mathrm{PSS}}^{\prime}(t)$ satisfies the homogeneous part of (3.3)

$$
\frac{\mathrm{d}}{\mathrm{d} t}(C(t) x)+G(t) x=0
$$


and assume the case of index-1 DAEs. For literature on the higher index cases we refer to [7]. Let

$$
\mathbf{S}(t):=\left\{z \in \mathbb{R}^{N} \mid\left(G(t)+\frac{\mathrm{d}}{\mathrm{d} t} C(t)\right) z \in \operatorname{Im}(C(t))\right\}, \quad \mathbf{N}(t):=\operatorname{Ker}(C(t))
$$

Then one has

$$
\mathbf{S}(t) \cap \mathbf{N}(t)=0, \quad \mathbf{S}(t) \oplus \mathbf{N}(t)=\mathbf{R}^{N},
$$

in the index- 1 case. We assume that $\mathbf{S}(t)$ is $m$-dimensional. There are $N$ independent solutions of the homogeneous problem: $u_{1}(t) e^{\mu_{1} t}, \ldots, u_{m}(t) e^{\mu_{m} t}, u_{m+1}(t), \ldots$, $u_{N}(t)$. The first $u_{1}(t), \ldots, u_{m}(t)$ are a basis of $\mathbf{S}(t)$; the last, $u_{m+1}(t), \ldots, u_{N}(t)$, are a basis of $\mathbf{N}(t)$. The $\mu_{1}, \ldots, \mu_{m}$ are so-called Floquet exponents; the $e^{\mu_{1} T}, \ldots$, $e^{\mu_{m} T}$ are Floquet multipliers. For a stable autonomous index-1 problem we can assume that $\mu_{1}=0$ and that $\operatorname{Re}\left(\mu_{i}\right)<0$ for $i=2, \ldots, m$. In this case we can choose $u_{1}(t)=x_{\mathrm{PSS}}^{\prime}(t)$.

The homogeneous adjoint (or dual) system corresponding to (3.3) is

$$
C^{\top}(t) \frac{\mathrm{d}}{\mathrm{dt}} y-G^{\top}(t) y=0
$$

Similar to the not-adjoint case we introduce

$$
\mathbf{S}^{\top}(t):=\left\{z \in \mathbb{R}^{n} \mid G^{\top}(t) z \in \operatorname{Im}\left(C^{\top}(t)\right)\right\}, \quad \mathbf{N}^{\top}(t):=\operatorname{Ker}\left(C^{\top}(t)\right),
$$

with the properties

$$
\mathbf{S}^{\top}(t) \cap \mathbf{N}^{\top}(t)=0, \quad \mathbf{S}^{\top}(t) \oplus \mathbf{N}^{\top}(t)=\mathbb{R}^{N} .
$$

Also $\mathbf{S}^{\top}$ is $m$-dimensional. The adjoint problem has $N$ independent solutions: $v_{1}(t) e^{-\mu_{1} t}, \ldots, v_{m}(t) e^{-\mu_{m} t}, v_{m+1}(t), \ldots, v_{N}(t)$, where $v_{1}(t), \ldots, v_{m}(t)$ are a basis of $\mathbf{S}^{\top}(t)$ and the last, $v_{m+1}(t), \ldots, v_{N}(t)$, are a basis of $\mathbf{N}^{\top}(t)$.

It easy to verify that if $x$ and $y$ are solutions of (3.3) and (3.15), respectively, the inner-products $y^{\top}(t) C(t) x(t)$ are constant, thus $y^{\top}(t) C(t) x(t)=y^{\top}(0) C(0) x(0)$, for all $t \geq 0$. More specifically, the bases $u_{1}(t), \ldots, u_{N}(t)$ and $v_{1}(t), \ldots, v_{N}(t)$ can be chosen such that, the $N \times N$ matrix $U(t)$ with as columns the $u_{i}(t)$ and the $N \times N$ matrix $V(t)$ with as rows the $v_{i}(t)$ satisfy a bi-orthogonality relation w.r.t. $C(t)$ and a nearly one w.r.t. $G(t)$

$$
\text { (3.21) } V(t) C(t) U(t)=\left(\begin{array}{cc}
I_{m} & 0 \\
0 & 0
\end{array}\right), \quad V(t) G(t) U(t)=\left(\begin{array}{cc}
J_{m}^{1} & 0 \\
J_{m}^{2} & J_{m}^{3}
\end{array}\right) .
$$

Here $I_{m}$ is an $m \times m$ identity matrix. $J_{m}^{1}$ is a $m \times m$ block matrix. $J_{m}^{2}$ and $J_{m}^{3}$ are suitable block matrices.

The monodromy matrix is the state-transition matrix after one period, i.e. $\Phi(T)=$ $U(T) D(T) V(0) C(0)$, where $D(T)=\operatorname{diag}\left(e^{\mu_{1} T}, \ldots, e^{\mu_{m} T}, 0, \ldots, 0\right)$ (this matrix arises in natural way when one considers shooting methods or applies Floquet theory to analyze stability of a limit cycle). Because of the periodicity of the $u_{i}$, we see that the $u_{i}(0)$, for $i=1, \ldots, m$, are eigenvectors of the monodromy matrix with corresponding eigenvalues $e^{\mu_{i} T}$, and that the remaining $u_{i}(0)$, for $i=m+1, \ldots, N$, are eigenvectors for the $(N-(m-1))$-fold eigenvalue 0 . Similar 
to the not-adjoint case, the $v_{i}(0)$ are eigenvectors of the associated monodromy $\operatorname{matrix} \Psi(T)=V^{\top}(T) D(-T) U^{\top}(0) C^{\top}(0)$.

3.2.2. Phase NOISE by NON-LINEAR PERTURbation analysis We will take $u_{1}(t)=x_{\text {PSS }}^{\prime}(t)$. Here $u_{1}$ is the tangent to the orbit. Let $\alpha(t)$ be a (sufficiently smooth) phase- or time-shift function and let $s=t+\alpha(t)$ be the shifted time. If $x_{\mathrm{PSS}}(t)$ is the PSS solution of (2.6) then the phase-shifted function $y(t) \equiv$ $x_{\mathrm{PSS}}(s)=x_{\mathrm{PSS}}(t+\alpha(t))$ satisfies

$$
\begin{aligned}
\frac{\mathrm{d}}{\mathrm{d} t} q(y)+j(y) & =\frac{\mathrm{d}}{\mathrm{d} s} q\left(x_{\mathrm{PSS}}(s)\right) \cdot \frac{\mathrm{d} s}{\mathrm{~d} t}+j\left(x_{\mathrm{PSS}}(s)\right) \\
& =\frac{\mathrm{d}}{\mathrm{d} x_{\mathrm{PSS}}} q\left(x_{\mathrm{PSS}}(s)\right) \frac{\mathrm{d} x_{\mathrm{PSS}}}{\mathrm{d} s} \alpha^{\prime}(t) \\
& =C(t+\alpha(t)) u_{1}(t+\alpha(t)) \alpha^{\prime}(t) .
\end{aligned}
$$

Hence, the phase shifted function $y$ satisfies a perturbed DAE in which the righthand side has a particular form.

We now consider perturbations of the form $B(x(t)) b(t)$ (cf. also (3.3)) to the original DAE (2.6)

$$
\frac{\mathrm{d}}{\mathrm{d} t} q(x)+j(x)+B(x(t)) b(t)=0,
$$

and express $B(x(t+\alpha(t))) b(t)$ into its components using the basis $\left\{C(t+\alpha(t)) u_{1}(t+\right.$ $\alpha(t)), \ldots, C(t+\alpha(t)) u_{m}(t+\alpha(t)), G(t+\alpha(t)) u_{m+1}(t+\alpha(t)), \ldots, G(t+\alpha(t)) u_{N}(t+$ $\alpha(t))\}$

$$
\begin{aligned}
B(x(t+\alpha(t))) b(t)= & \sum_{i=1}^{m} c_{i}(x, \alpha(t), t) C(t+\alpha(t)) u_{i}(t+\alpha(t)) \\
& +\sum_{i=m+1}^{N} c_{i}(x, \alpha(t), t) G(t+\alpha(t)) u_{i}(t+\alpha(t)) \\
c_{i}(x, \alpha(t), t)= & \tilde{v}_{i}(t+\alpha(t)) b(t), \text { where } \\
\tilde{v}_{i}(t)= & v_{i}^{\top}(t) B(x(t)) .
\end{aligned}
$$

Here the scalar functions $\tilde{v}_{i}(t)$ are periodical in $t$ with period $T$.

The first component of $B(x(t+\alpha(t))) b(t)$ will be used to determine $\alpha(t)$. We define $\alpha(t)$ to satisfy the non-linear, scalar, differential equation

$$
\begin{aligned}
\alpha^{\prime}(t) & =-v_{1}^{\top}(t+\alpha(t)) B\left(x_{\operatorname{PSS}}(t+\alpha(t))\right) b(t), \quad \alpha(0)=0 \\
& =-\tilde{v}_{1}(t+\alpha(t)) b(t), \quad \alpha(0)=0 .
\end{aligned}
$$

(See also $[11,12]$ where a first start was made to treat the phase noise problem in the time domain.) Note that if $b(t)=0$, for $t \geq t_{0}$, then $\alpha$ becomes a constant phase shift, and the phase shifted function $y(t)$ solves exactly (3.23) for $t \geq t_{0}$. Also note that the explicit form of $B(x(t))$ is never used. If we take $B(x(t)) b(t)=$ 
$C(x(t)) u_{1}(t) b(t)$, with $b(t)$ scalar, bi-orthogonality implies

$$
\begin{aligned}
\alpha^{\prime}(t) & =-b(t), \alpha(0)=0, \text { hence } \\
\alpha(t) & =-\int_{0}^{t} b(t) \mathrm{d} t .
\end{aligned}
$$

Clearly, for this case, non-linearity does not occur. For $b(t)=\sin (\omega t)$ we find $\alpha(t)=\frac{1}{\omega} \cos (\omega t)$, which shows a $1 / \omega$ behaviour. In general, however, (3.28) is non-linear and this effect is really needed when modelling Phase Locked Loop oscillators. A linear model, like in [8], is of limited use. Also even for small $b$, the phase shift function $\alpha(t)$ may increase with time (Clearly, if $N=1, B \equiv 1, b(t) \equiv \varepsilon$, and $v_{1}^{\top}(t) \equiv \kappa$, then $\left.\alpha(t)=\kappa \varepsilon t\right)$.

To find $\alpha$, we clearly have to know $v_{1}$. In [6] this crucial vector is called Perturbation Projection Vector, or PPV. It represents a transfer between the perturbation of the DAE and the resulting phase shift.

In [4] $v_{1}$ is determined by performing first an eigenvalue/eigenvector analysis of the monodromy matrix of the adjoint problem to obtain $v_{1}(0)$, followed by time integration (backward in time). To discern the proper initial value $v_{1}(0)$ from other eigenvectors that have eigenvalues close to 0 , one can exploit the bi-orthogonality relation (3.21), because $v_{1}(0)$ must have a non-trivial $C$-inner-product with $u_{1}(0)$. Another, direct, approach is found in [6]. It nicely fits a Finite Difference Method approach and again exploits the bi-orthogonality relation (3.21) in an elegant way. For deterministic perturbations one has to integrate (3.28). Because of this action in the time domain, all Fourier components of $n(t)$ are treated in a combined way. However, for stochastic noise, such a detail is not necessary. In $[4,5]$ expressions for the power due to the noise are derived that depend on the asymptotic behaviour (i.e. for large $t$ ) of the variance $\operatorname{var}[\alpha(t)]$. The authors derive power spectrum expressions that depend on the Fourier components of the PSS solution $x_{P S S}$, on the DC component of $v_{1}(t)$, and on the power spectrum of $b$. The power of the $j$-th harmonic of $x_{\text {PSS }}$ is preserved in the power of the 'asymptotic' $j$-th harmonic of $y$ (i.e. the shifted $x_{\mathrm{PSS}}$ ). Consequently, by summing over $j$, we see that also the total power is preserved. In case of stochastically independent sources, one can determine the contributions to the variance $\operatorname{var}[\alpha(t)]$ quite elegantly.

More specifically, let

$$
x_{\mathrm{PSS}}(t)=\sum_{j=-\infty}^{\infty} X_{j} e^{i \omega_{j} t},
$$

where $\omega_{j}=2 \pi j f_{\mathrm{osc}}=2 \pi j / T$. The stationary autocorrelation of the phase-shifted function $y(t)$ can be expressed as an asymptotic stochastic expectation of the autocorrelation

$$
\begin{aligned}
R_{y}(\tau) & \equiv \lim _{t \rightarrow \infty} R_{y}(t, \tau), \quad \text { where } \\
R_{y}(t, \tau) & =E\left[y(t) y^{*}(t+\tau)\right] .
\end{aligned}
$$


The spectral density that corresponds to $R_{y}(\tau)$ can be expressed as

$$
S_{y}(\omega)=\sum_{j=-\infty}^{\infty} X_{j} X_{j}^{*} S_{j}\left(\omega+\omega_{j}\right),
$$

where each $S_{j}$ satisfies $\int_{\infty}^{\infty} S_{j}(2 \pi f) \mathrm{d} f=1$. The expressions for $S_{j}$ can be related to the spectra for the local noise sources $b(t)$ and involve the DC component of the PPV transfer $\tilde{v}_{1}(t)[4,5]$.

In practice, noise criteria are based on the the two-sided phase noise power spectral density in $V^{2} / H z$

$$
\begin{aligned}
S_{\mathrm{pp}}^{k}(\omega) & =S_{y}\left(\omega+\omega_{k}\right) \\
& =\sum_{j=-\infty}^{\infty} X_{j} X_{j}^{*} S_{j}\left(\omega+\omega_{k}+\omega_{j}\right) \\
& =\sum_{j=-\infty}^{\infty} X_{j} X_{j}^{*} S_{j}\left(\omega+\omega_{k+j}\right)
\end{aligned}
$$

Summation is performed for all $j$ for which both $j$ and $j+k$ are in the range of calculated harmonics. Other well-known representations are the power spectral density in $d B / H z$

$$
d B S_{\mathrm{pp}}^{k}=10 \log _{10}\left(S_{\mathrm{pp}}^{k}\right)
$$

and the power spectral density with respect to the carrier in $d B c / H z$

$$
d B c S_{\mathrm{pp}}^{k}=10 \log _{10}\left(\frac{S_{\mathrm{pp}}^{k}}{\left|X_{k}\right|^{2}}\right) .
$$

3.2.3. Orbital Deviation The solution $x$ of (3.23) may be written as $x(t)=$ $y(t)+z(t)$, where $z$ is the orbital deviation function, which can be analysed by a proper linear perturbation analysis (but with linearised equations which now have non-periodic coefficients!). Because $n$ also affects the phase-shifted function, around which one linearises to study the orbital devations, there is no simple summation formula known for cumulative noise contributions. In [4,5] it is argued that, in first order, $z(t)$ satisfies $\|z\|_{\infty}<$ Const. $\|b\|_{\infty}$ (and even $z(t) \rightarrow 0(t \rightarrow \infty)$ ). We will assume that $b$ is a scalar function, for simplicity. Then, in first order, $z$ satisfies a linear differential equation

$$
\frac{\mathrm{d}}{\mathrm{d} t}\left[C_{y}(t) z(t)\right]+\left[G_{y}(t)+B_{y}(t) b(t)\right] z(t)+B_{1}(y(t)) b(t)=0
$$

where

$$
\begin{aligned}
& C_{y}(t)=\frac{\mathrm{d}}{\mathrm{d} y} q(y(t)), \quad G_{y}(t)=\frac{\mathrm{d}}{\mathrm{d} y} j(y(t)) \\
& B_{y}(t)=\frac{\mathrm{d}}{\mathrm{d} y} B(y(t)), \quad B_{1}(y(t)) b(t)=\left[B(y(t))-B_{2}(y(t))\right] b(t), \\
& B_{2}(y(t))=v_{1}^{\top}(t+\alpha(t)) B(y(t)) C(t+\alpha(t)) u_{1}(t+\alpha(t)),
\end{aligned}
$$


where $B_{2}$ is the component of $B$ in the $C$-inner-product along $u_{1}$. If we write $z(t)=\tilde{z}(t+\alpha(t))=\tilde{z}(s),(3.36)$ can be written more in terms of $s$

$$
\begin{aligned}
&-\frac{\mathrm{d}}{\mathrm{d} s}\left[C\left(x_{\mathrm{PSS}}(s)\right) \tilde{z}(s)\right] v_{1}^{\top}(s) B\left(x_{\mathrm{PSS}}(s)\right) b(t) \\
&+\left[G\left(x_{\mathrm{PSS}}(s)\right)+B_{x}\left(x_{\mathrm{PSS}}(s)\right) b(t)\right] \tilde{z}(s)+B_{1}\left(x_{\mathrm{PSS}}(s)\right) b(t)=0 .
\end{aligned}
$$

We note that if $b(t) \equiv 0$ for $t \geq t_{0}, \tilde{z}(s)=0$ for some $s \geq s_{0}$ (assuming that $G\left(x_{\mathrm{PSS}}(s)\right)$ has full rank). If $b(t) \equiv c$ for some constant $c$ all coefficients in (3.37) are periodic in $s$.

For small $b$ this linear differential equation in $\tilde{z}(s)$ is singularly perturbed and inhomogeneous. The coefficients depend on $b$, which implies that the transfer function depends on the (group of) sources as well.

If we assume that $\tilde{z}(s)$ and $\tilde{z}^{\prime}(s)$ are both of order $\mathcal{O}(\| b||),(3.37)$ implies the first order linear system

$$
\begin{aligned}
G\left(x_{\mathrm{PSS}}(s)\right) \tilde{z}(s) & =-B_{1}\left(x_{\mathrm{PSS}}(s)\right) b(t), \\
& =-\left[B\left(x_{\mathrm{PSS}}(s)\right)-B_{2}\left(x_{\mathrm{PSS}}(s)\right)\right] b(t), \\
& =-\left[B\left(x_{\mathrm{PSS}}(s)\right)-v_{1}^{\top}(s) B\left(x_{\mathrm{PSS}}(s)\right) C\left(x_{\mathrm{PSS}}(s)\right) u_{1}(s)\right] b(t), \\
& =-\left[B\left(x_{\mathrm{PSS}}(s)\right)-v_{1}^{\top}(s) B\left(x_{\mathrm{PSS}}(s)\right) C\left(x_{\mathrm{PSS}}(s)\right) u_{1}(s)\right] \tilde{b}(s),
\end{aligned}
$$

where $\tilde{b}(s)$ is defined by $\tilde{b}(s)=b(t)$. We note that for the system (3.38), at the right-hand side, the term between the brackets can completely be determined in the $s$-variables; note that all quantities are periodic in $s$. The factor $\tilde{b}(s)$ has to be determined in the frequency domain.

\section{Simple RLC oscillator example}

We consider a simple RLC oscillator to simulate phase noise. The schematic is shown in Fig. 1 (Top-Left). Here $\mathrm{L}_{0}=1 \mu \mathrm{H}, \mathrm{C}_{1}=1 \mu \mathrm{F}, \mathrm{E}_{0}=5.0 \mathrm{~V}, \mathrm{R}_{3}=1 \mathrm{~m} \Omega$, $\mathrm{R}_{l}=50 \Omega, \mathrm{R}_{s}=50 \Omega$. An oscillator is "controlledly unstable". The instability is caused by the negative resistance, $\mathrm{R}_{0}: i=0.4 \tanh (-5 v)$, the nonlinearity provides control in excluding unlimited growth. The value 0.4 determines the height of the oscillation amplitude and the $\tanh (-5 v)$ factor determines the time moment when the oscillation will start.

The basic RLC-components are $\mathrm{L}=\mathrm{L}_{0}, \mathrm{C}=\mathrm{C}_{1}$ and $\mathrm{R}=\mathrm{R}_{3}$. The series resistor $\mathrm{R}_{3}$ models the effect of a non-ideal coil. The resonant angular frequency $\omega_{0}$ of an RLC oscillator, with a non-ideal coil, can be determined by $\omega_{0}=\frac{1}{\sqrt{L C}} \sqrt{1-\frac{R^{2} C}{4 L}} \approx$ $\frac{1}{\sqrt{L C}}=10^{6}$ because $\frac{R^{2} C}{4 L} \ll 1$. This corresponds to a frequency $f_{0}=159.24 \mathrm{kHz}$. In general one will estimate the frequency by a pole-zero analysis, or by applying the Kurokawa condition [16], or from an initial transient analysis, or as part of a PSS analysis $[9,10]$. The intended oscillation can be observed at the nodal voltage $v_{n}(1)$. From the PSS analysis one derives $f_{0}=159.11 \mathrm{kHz}$ as PSS frequency. In Fig. 1 results are shown. At the Top-Right the amplitude of the Fourier Transform 

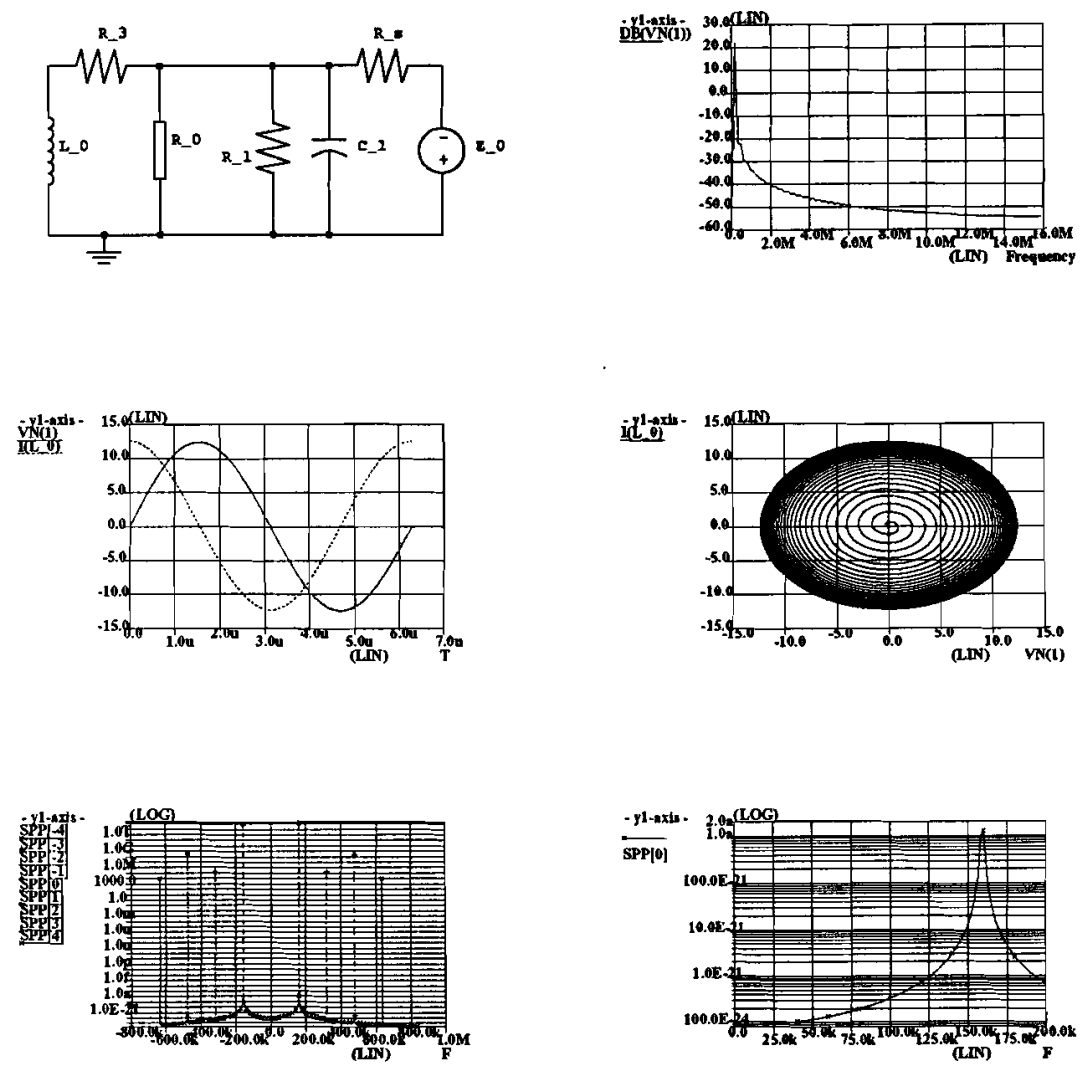

Figure 1. RLC Oscillator: Top-Left: Schematic, Top-Right: Amplitude of Fourier Transform of $v_{n}^{\text {PSS }}(1)$, Center-Left: Time signal of $v_{n}^{\mathrm{PSS}}(1)$, Center-Right: $i\left(L_{0}\right)$ is plotted against $v_{n}(1)$, BottomLeft: Output phase noise voltage spectrum $S_{\mathrm{pp}}^{k}$, Bottom-Right: Output phase noise voltage spectrum $S_{\mathrm{pp}}^{0}$.

of $v_{n}(1)$ is shown, which has magnitude about $21.9 \mathrm{dBV}$, which compares well to the amplitude of the PSS time signal, shown at Center-Left. In the Center-Right figure, the current $i\left(L_{0}\right)$ is plotted against $v_{n}(1)$. Sweeping the "noise" frequency $f_{\mathrm{sw}}$ from 0 to $200 \mathrm{kHz}$ in a Periodic $\mathrm{AC}$ analysis leads to output at frequencies $f_{\text {out }}=f_{\mathrm{sw}}+k f_{0}$. At the Bottom-Right the output phase noise $S_{\mathrm{pp}}^{k}$ voltage spectrum in $\mathrm{V}^{2} / \mathrm{Hz}$ is displayed for the whole circuit. $S_{\mathrm{pp}}^{0}$ gives the phase noise around 
the oscillation frequency. The smaller the integrated area, the less phase noise is present.

\section{References}

[1] W. Anzill, F.X. Kärtner, P. Russer: Simulation of the phase noise of oscillators in the frequency domain, Int. J. Electron. Commun. (AEÜ) 48-1 (1994), 45-50.

[2] W. Bomhof, H.A. van der Vorst (2001): A parallelizable GMRES-type method for pcyclic matrices with applications in circuit simulation, in U. van Rienen, M. Günther, D. Hecht (Eds): Scientific computing in electrical engineering, Proc. SCEE-2000, Warnemünde, Springer, Berlin (2001), 293-300.

[3] A. Demir, A. Sangiovanni-Vincentelli: Analysis and Simulation of noise in nonlinear electronic circuits and systems, Kluwer Academic Publ., Boston, USA, 1998.

[4] A. Demir, A. Mehrotra, J. Roychowdhury: Phase noise in oscillators: a unifying theory and numerical methods for characterisation, IEEE Trans. on Circuits and Systems - I: Fund. Theory and Applics. 47-5 (2000), 655-674.

[5] A. Demir: Phase noise in oscillators: DAEs and coloured noise sources, Proc. ICCAD'98, Int. Conf. on Computer Aided Design, San Jose, CA, USA (1998), 170-177.

[6] A. Demir, D. Long, J. Roychowdhury: Computing phase noise eigenfunctions directly from Harmonic Balance/Shooting matrices, Proc. ICCAD 2000, Int. Conf. on Computer Aided Design, San Jose, CA, USA (2000), 283-288.

[7] M. Günther, U. Feldmann, J. ter Maten: Discretization of circuit problems, to appear in Numerical Analysis of Electromagnetics, Series Handbook of Numerical Analysis, Elsevier Science.

[8] A. Hajimiri, T.H. Lee: A general theory of phase noise in electrical oscillators, IEEE J. Solid-State Circ., 33-2 (1998), 179-194.

[9] S.H.M.J. Houben, E.J.W. ter Maten, J.M. Maubach, J.M.F. Peters: Novel timedomain methods for free-running oscillators, In: V. Porra, M. Valtonen, I. Hartimo, M. Ilmonen, O. Simula, T. Veijola (Eds): ECCTD'01 - Proceedings of the 15TH European Conference on Circuit Theory and Design, Helsinki University of Technology (ISBN 951-22-5571-5), Finland (2001), III-393 - III-396.

[10] S.H.M.J. Houben: Time-domain simulation techniques for finding the PSS of electric oscillators, these Proceedings.

[11] F.X. Kärtner: Untersuchung des Rauschverhaltens von Oszillatoren, PhD-Thesis TU München, 1989.

[12] F.X. Kärtner: Analysis of white and $f^{-\alpha}$ noise in electrical oscillators, Int. J. Circuit Theory Appl. 18 (1990), 485-519.

[13] T.A.M. Kevenaar: Periodic Steady State Analysis using Shooting and WaveformNewton, Int. J. Circuit Theory and Applics. 22 (1994), 51-60.

[14] R. Lamour, R. März, R. Winkler: How Floquet Theory applies to Index 1 differential algebraic equations, J. of Math. Analysis and Applics 217 (1998), 372-394.

[15] R. Lamour: Floquet-Theory for differential-algebraic equations (DAE), ZAMM 78-3 (1998), S989-S990. 
[16] S. Lampe, H.G. Brachtendorf, E.J.W. ter Maten, S.P. Onneweer, R. Laur: Robust limit cycle calculations of oscillators, In U. van Rienen, M. Günther, D. Hecht (Eds.): Scientific computing in electrical engineering, Proc. SCEE-2000, Warnemünde/Rostock, LNCSE 18, Springer Verlag, Berlin (2001), 233-240.

[17] E.J.W. ter Maten: Numerical methods for frequency domain analysis of electronic circuits, Surv. Math. Ind. 8 (1999), 171-185.

[18] K. Mayaram, D.C. Lee, S. Moinian, D. Rich, J. Roychowdhury: Overview of computer-aided analysis tools for RFIC: algorithms, features, and limitations, IEEE 1997 Custom Integrated Circuit Conference, Santa Clara, CA, USA (1997), 505-512.

[19] M. Okumura, T. Sugawara, H. Tanimoto: An efficient small signal frequency analysis method for nonlinear circuits with two frequency excitations, IEEE Trans. of Comp.Aided Design of Integrated Circuits and Systems 9-3 (1990), 225-235.

[20] M. Okumura, H. Tanimoto, T. Itakura, T. Sugawara: Numerical noise analysis for nonlinear circuits with a periodic large signal excitation including cyclostationary noise sources, IEEE Trans. on Circuits and Systems - I: Fund. Theory and Applics. 40-9 (1993), 581-590.

[21] R. Telichevesky, K.S. Kundert, J.K. White: Efficient Steady-State Analysis based on matrix-free Krylov-Subspace methods, Proc. DAC'95, 1995.

[22] R. Telichevesky, K. Kundert: Efficient $A C$ and noise analysis of two-tone $R F$ circuits, Proc. DAC'96, Las Vegas, 1996.

\section{Acknowledgment}

We thank all involved in organising the inspiring 2001 Oberwolfach Meeting 48 on Modellierung, Simulation und Optimierung intergrierter Schaltkreise.

Joint address: Philips Research Laboratories and Eindhoven University of Technology, c/o Prof. Holstlaan 4, Bldng WAY, NL-5656 AA Eindhoven, The Netherlands E-mail address: Jan.ter. Maten@philips.com 